\title{
Relaxational trajectories: global approximations
}

\author{
Alexander N.Gorban ${ }^{\text {a }}$, Iliya V. Karlin ${ }^{\text {a, } b, *}$, Vladimir B. Zmievskii ${ }^{a}$ \\ T.F. Nonnenmacher ${ }^{b}$, \\ ${ }^{a}$ Computing Center, Russian Academy of Sciences, Krasnoyarsk 660036, Russian Federation \\ ${ }^{\mathrm{b}}$ Department of Mathematical Physics, University of Ulm, Ulm D-89069, Germany
}

Received 15 November 1995; revised 28 February 1996

\begin{abstract}
The paper intends to fill the gap of analytic approximate methods for non-linear spaceindependent dissipative systems equipped with the entropy functional. The key point of the analysis is an upper limiting state in the beginning of the relaxation. Extremal properties of this state are described and explicit estimations are derived. This limiting state is used to construct explicit approximations of the phase trajectories. Special attention is paid to accomplish positivity, smoothness and the entropy growth along the approximate trajectories. The method is tested for the space-independent Boltzmann equation with various collisional mechanisms.
\end{abstract}

\section{Introduction}

When a relaxing system is considered, it is common to distinguish between the three subsequent regimes on the way from an initial non-equilibrium state $f_{0}(\Gamma)$ to the final equilibrium state $f^{0}(\Gamma)$, where $\Gamma$ is the phase variable: the early-time relaxation immediately after the system leaves the initial state $f_{0}$, the intermediate regime, and the final regression to the equilibrium state $f^{0}$. This model picture is only approximate and it is most relevant to gases. The early-time relaxation occurs in a few first collisions of the molecules and can be singled out from the whole relaxational process to be investigated separately [1].

Considering the beginning of a relaxation, we may expect that it is dominated by a rate of processes in the initial state. In the case of a gas, in particular, this rate $Q_{0}$ is given by a collision integral $Q(f)$ computed in the state $f_{0}: Q_{0}=Q\left(f_{0}\right)$. The latter expression can be regarded as a known function of the phase variable, $Q_{0}(\Gamma)$. In other

\footnotetext{
* Corresponding author; Present address: Department of Mathematical Physics, University of Ulm, Ulm D-89069, Germany; fax: (0731)502-3003: e-mail: kar@physik.uni-ulm.de.
} 
words our expectation is that states which the systems pass through in the beginning are close to those on a ray, $f(\Gamma, a)$ :

$$
f(\Gamma, a)=f_{0}(\Gamma)+a Q_{0}(\Gamma),
$$

where $a \geqslant 0$ is a scalar variable (throughout the paper we use dimensionless variables). It is clear that such an approximation can be valid only if $a$ "is not too large". On the other hand, nothing tells us ultimately that $a$ must be "strictly infinitesimal" to get at least a moderate approximation. In general, this consideration can be relevant if the parameter $a$ in Eq. (1) does not exceed a certain upper limit $a^{*}$.

In this paper we give an answer to the following question: what is the upper limiting state $f^{*}$ the system cannot overcome when driven with the initial rate $Q_{0}$ ? As long as we can consider $Q_{0}$ as the dominant direction in the early-time relaxation, the answer amounts to an upper estimate of the parameter $a$ in Eq. (1), and thus the limiting state $f^{*}$ is

$$
f^{*}(\Gamma)=f\left(\Gamma, a^{*}\right)=f_{0}(\Gamma)+a^{*} Q_{0}(\Gamma),
$$

where the value $a^{*}$ is the subject of the analysis to be performed.

Our approach will be based on the following consideration. Denote by $S(f)$ the entropy of a state $f(\Gamma)$ and by $S(a)$ its value in the state $f(\Gamma, a)$ of Eq. (1). A state $f\left(\Gamma, a^{\prime}\right)$ can be regarded accessible from the initial state $f(\Gamma, 0)=f_{0}(\Gamma)$ in the course of the $Q_{0}$-dominated dynamics, if and only if the function $S(a)$ increases with an increase in the variable $a$ from 0 to $a^{\prime}$. The upper limiting value $a^{*}$ is thus characterized by the following two properties:

1. $S(a)$ increases as $a$ increases from 0 to $a^{*}$.

2. $S(a)$ decreases as $a$ overcomes $a^{*}$.

Assuming the usual convexity properties of the entropy, we conclude that the state $f\left(\Gamma, a^{*}\right)$ with these properties is unique.

The paper is organized as follows. In Section 2, we derive an equation for the limiting state $f\left(\Gamma, a^{*}\right)$ in two ways: firstly, as a direct conjecture of the two properties just mentioned and, secondly, as an equilibrium state of an appropriately chosen model of the $Q_{0}$-dominated relaxation. Next we introduce a method to obtain the explicit estimations of the function $f\left(\Gamma, a^{*}\right)$ (details are given in Appendix A). With this, we get the answer to the question posed above.

The derivation of the state $f\left(\Gamma, a^{*}\right)$ plays the key role in Section 3. There we aim at constructing explicit approximations to phase trajectories of a given space-independent kinetic equation: namely, we construct a function $f(\Gamma, a)$, where the parameter $a$ spans a segment $[0,1]$, and which fits the following conditions:

1. $f(\Gamma, 0)=f_{0}(\Gamma)$

2. $f(\Gamma, 1)=f^{0}(\Gamma)$;

3. $f(\Gamma, a)$ is a non-negative function of $\Gamma$ for each $a$;

4. $C(a)=C(f(a))=$ const, where $C(f)$ are linear conserved quantities;

5. $S(a)=S(f(a))$ is a monotonically increasing function of $a$;

6. $\partial f(\Gamma, a) /\left.\partial a\right|_{a=0}=k Q_{0}(\Gamma)$, where $k>0$. 
With this, $f(\Gamma, a)$ is thought of as a path from the initial state $f_{0}$ to the equilibrium state $f^{0}$ (conditions 1 and 2), all states of the path make physical sense (condition 3 ), conserved quantities do not change, and the entropy monotonically increases along the path (conditions 4 and 5). Lastly, condition 6 requires the path to be tangent to the exact phase trajectory in their common initial state $f_{0}$. A function $f(\Gamma, a)$ with the properties 1-6 is, of course, not unique but a construction of a definite example occurs to be a non-trivial task. Indeed, the major difficulty is that the tangency condition 6 is not directly compatible with the rest of the conditions.

The simplest function with the properties 1-6, and which depends smoothly on $a$, is constructed explicitly in the Section 3 (details of the procedure are given in Appendix B). We also discuss the question of the time dependence of $f(\Gamma, a(t))$. In Section 4 , the method is applied to the space-independent non-linear Boltzmann equation for several collisional mechanisms. In particular, we compare our approximations with the known BKW-mode [2,3] for the Maxwellian molecules, and with the solutions to the two-dimensional very hard particles model (VHP) [4,5]. Results are discussed in the Section 5.

Prior to proceeding further, it is worthwhile to give here a brief comment on the status of the approximate phase trajectories considered in Section 3. It is well known that the space-independent problem for dissipative kinetic equations is one of the most developed branches of kinetic theory, primarily, with respect to existence and uniqueness theorems [6-8], as well as to the exact treatment of specific models $[9,10]$. On the other hand, there exists a gap of approximate analytic methods in this problem. This is not surprising because most of the techniques of the kinetic theory [1] are based on a small parameter expansion, and this is not the case of the space-independent relaxation. Our consideration intends to make the first step to fill this gap. Indeed, as the examples demonstrate, the smooth approximations $f(a, \Gamma)$ of Section 3 provide a reasonable (and simple) approximation to the exact trajectory.

Moreover, these functions serve for the initial approximation in an iterative method of constructing the exact trajectories for the dissipative systems [11] (this method, in turn, is based on a more general consideration of the paper [12]). We will give additional comments on this iterative method in Section 5 and provide an illustration of the correction in Section 4, leaving, however, a detailed discussion for a further publication.

\section{Extremal properties of the limiting state}

Let us first consider the equation for the limiting state $f\left(\Gamma, a^{*}\right)$ (Eq. (2)) in an informal way. The two features of the function $f\left(\Gamma, a^{*}\right)$ indicated above tell us that it is a state of the entropy maximum on the ray $f(\Gamma, a)(\mathrm{Eq} .(1))$. The extremum condition in this state reads

$$
\left.\int \frac{\partial f(\Gamma, a)}{\partial a} \cdot \frac{\delta S(f)}{\delta f}\right|_{f=f\left(\Gamma, a^{*}\right)} d \Gamma=0,
$$


where $\delta S / \delta f$ denotes a (functional) derivative of the entropy, taken in the state $f\left(\Gamma, a^{*}\right)$. For a particularly interesting case of $S_{B}(f)=-\int f(\Gamma) \ln f(\Gamma) d \Gamma$ (the Boltzmann entropy) and $\int Q(f) d \Gamma=0$ (conservation of the number of particles), Eq. (3) gives:

$$
\int Q_{0}(\Gamma) \ln \left\{f_{0}(\Gamma)+a^{*} Q_{0}(\Gamma)\right\} d \Gamma=0 .
$$

To avoid duplication of formulas and in view of the examples considered below, we will restrict our consideration to the latter case. A (unique) positive solution to Eq. (4) is the value $a^{*}$ which gives the desired upper estimate.

To derive Eq. (4) more formally, an explicit presentation is required for a model dynamics dominated by $Q_{0}$. Let us introduce a partition of the phase space into two domains, $\Gamma_{+}$and $\Gamma_{-}$, in such a way that the function $Q_{0}(\Gamma)$ is positive on $\Gamma_{+}$and negative on $\Gamma_{-}$, and so $Q_{0}(\Gamma)=Q_{0}^{+}(\Gamma)-Q_{0}^{-}(\Gamma)$, where both the functions $Q_{0}^{+}(\Gamma)$ and $Q_{0}^{-}(\Gamma)$ are positive and concentrated on $\Gamma_{+}$and $\Gamma_{-}$, respectively ${ }^{1}$. Let us consider the following relaxational equation:

$$
\partial_{t} f(\Gamma, t)=k_{1}\left(Q_{0}^{+}(\Gamma)-Q_{0}^{-}(\Gamma)\right)\left(w^{-}(f)-w^{+}(f)\right)
$$

where

$$
\begin{aligned}
& w^{-}(f)=\exp \left(\int_{\Gamma_{-}} Q_{0}^{-}(\Gamma) \ln f(\Gamma, t) d \Gamma\right), \\
& w^{+}(f)=\exp \left(\int_{\Gamma_{+}} Q_{0}^{+}(\Gamma) \ln f(\Gamma, t) d \Gamma\right),
\end{aligned}
$$

and $k_{1}>0$ is an arbitrary constant. When supplied with the initial condition $f(\Gamma, 0)=$ $f_{0}(\Gamma), \mathrm{Eq} .(5)$ has a formal solution of the form

$$
f(\Gamma, t)=f_{0}(\Gamma)+a(t) Q_{0}(\Gamma)
$$

provided that $a(t)$ is a solution to the ordinary differential equation

$$
\frac{d a}{d t}=k_{1}\left(w^{-}(a)-w^{+}(a)\right)
$$

with the initial condition $a(0)=0$.

The solution (7) describes a relaxation from the initial state $f_{0}$ to an equilibrium state $f^{*}$; as; $t$ tends to infinity. ${ }^{2}$ The entropy $S_{B}$ increases monotonically along this

\footnotetext{
'For the Boltzmann equation, this partition should not be confused with the natural representation of the collision integral as $\int w\left(\mathbf{v}_{1}^{\prime} \mathbf{v}^{\prime} \mid \mathbf{v}_{1} \mathbf{v}\right)\left(f^{\prime} f_{1}^{\prime}-f f_{1}\right) d \mathbf{v}_{1}^{\prime} d \mathbf{v}^{\prime} d \mathbf{v}_{1}$, see Section 4 .

${ }^{2}$ The equilibrium state $f^{*}$ of the model kinetic equation (5) is not the global equilibrium $f^{0}$.
} 
solution up to the value $S_{B}^{*}=S_{B}\left(f^{*}\right)$ in the state $f^{*}$. Substituting $f^{*}=f_{0}+a^{*} Q_{0}$ into the RHS of Eq.(5), we obtain an equation for the equilibrium state $f^{*}$ in the form

$$
w^{-}\left(a^{*}\right)=w^{+}\left(a^{*}\right)
$$

which is exactly Eq. (4). Note that the parameter $k_{1}$ in Eq. (5) does not affect the final result (4) since it is responsible only for the speed of the approach to the equilibrium state $f^{*}$ due to the dynamics (5) but not for the location of this state on the ray (1).

Let us discuss the idea behind the model dynamics presented by Eq. (5). As we disregard any changes of $Q$ in the beginning of the relaxation, the function $Q_{0}(\Gamma)$ represents a distinguished direction of relaxation in a space of states. Partition used corresponds then to a distinction of the gain $\left(\Gamma_{+}\right)$and of the loss $\left(\Gamma_{-}\right)$of the phase density, while the factors $w^{+}$and $w^{-}$in Eq. (6),

$$
w^{ \pm}(f) \sim \exp \left\{-\int_{\Gamma_{ \pm}} Q_{0}^{ \pm}(\Gamma) \frac{\delta S(f)}{\delta f(\Gamma)} d \Gamma\right\},
$$

give the rates of the gain and the loss in the current state $f$, respectively. Eq. (5) accounts for these processes in the familiar "gain minus loss" form, while the state $f^{*}$ corresponds to the balance of the gain and the loss (Eq. (8)). One can also observe a formal analogy of the structure of Eq. (5) to that of the so-called Marcelin-De Donder equations of chemical kinetics $[13,14]$.

Thus, the limiting state $f^{*}=f_{0}+a^{*} Q_{0}$ is described as the equilibrium state of the relaxational equation (5), and it is given by the solution to Eq. (4). Note that the parameter $a^{*}$ is correctly defined by Eq. (4), independently of the partition introduced in Eq. (5). The existence of the model relaxational equation (5) guarantees that $f^{*}$ is the physical state represented by a non-negative distribution function.

To complete the analysis, we have to learn to solve the one-dimensional non-linear equation (4). In general, a method of successive approximation is required to get the solution $a^{*}$ as a limit of the sequence $a_{1}^{*}, a_{2}^{*}, \ldots$. Care should be taken to get all the approximations $a_{i}^{*}$ not greater than the unknown exact value $a^{*}$, since the states $f(a, \Gamma)$ with $a \leqslant a^{*}$ are only relevant. Moreover, what one actually needs in computations is some definite approximation $a_{1}^{*} \leqslant a^{*}$. In Appendix A, a relevant method is developed which is based on the partition of $Q_{0}$ introduced above. In particular, the first approximate $a_{1}^{*}$ reads

$$
a_{1}^{*}=\frac{1-\exp \left\{-\sigma_{0} / q\right\}}{\alpha+\beta \exp \left\{-\sigma_{0} / q\right\}},
$$


where $q, \sigma_{0}, \alpha$, and $\beta$ are numerical coefficients:

$$
\begin{aligned}
& \sigma_{0}=-\int Q_{0}(\Gamma) \ln f_{0}(\Gamma) d \Gamma, \\
& q=\int_{\Gamma_{+}} Q_{0}^{+}(\Gamma) d \Gamma=\int Q_{\Gamma_{-}}^{-}(\Gamma) d \Gamma, \\
& \alpha=\sup _{\Gamma \in \Gamma-Q_{-}} \frac{Q_{0}^{-}(\Gamma)}{f_{0}(\Gamma)} \\
& \beta=\int_{\Gamma_{+}} \frac{\left(Q_{0}^{+}(\Gamma)\right)^{2}}{q f_{0}(\Gamma)} d \Gamma .
\end{aligned}
$$

In these expressions, $\sigma_{0}$ is the entropy production in the initial state, $q$ is the normalizing factor, and $\alpha$ and $\beta$ reflect the maximal loss and the total gain of the phase density in the initial state, respectively. Finiteness of the parameters collected in (10) gives a restriction on the initial state $f_{0}$ for the estimate (9) to be applied.

\section{Approximate phase trajectories}

In this section we will demonstrate how one can utilize the states $f^{*}$ of Eq. (2) in the problem of constructing the approximate phase trajectories of the space-independent relaxational equations:

$$
\partial_{t} f(\Gamma, t)=Q(f)
$$

Here $Q(f)$ is a kinetic operator. We assume that Eq.(11) describes a relaxation to the equilibrium state $f^{0}(\Gamma)$, and the entropy $S_{B}(f)$ increases monotonically along the solutions. Further, let $c_{1}(\Gamma), \ldots, c_{k}(\Gamma)$ be the conserving quantities, i.e. $\int c_{i} Q(f) d \Gamma=$ 0. Then $C_{i}(f)=\int c_{i} f d \Gamma$ conserves along a solution, and $\ln f^{0}(\Gamma)=\sum_{1}^{k} a_{i} c_{i}(\Gamma)$, where $a_{i}$ are some numbers. A standard example of Eq. (11) is the space-independent Boltzmann equation to be considered in Section 4 .

Let $f(\Gamma, t)$ be the solution of Eq. (11) with the initial condition $f(\Gamma, 0)=f_{0}(\Gamma)$. The phase trajectory of this solution can be represented as a function $f(\Gamma, a)$, where $a$ varies from 0 to 1 . For each $a$, the function $f(\Gamma, a)$ is a non-negative function of $\Gamma$, and

$$
\begin{aligned}
& f(\Gamma, 0)=f_{0}(\Gamma), \quad f(\Gamma, 1)=f^{0}(\Gamma) \\
& \int c_{i}(\Gamma) f(\Gamma, a) d \Gamma=\text { const },\left.\quad \partial_{a} f(\Gamma, a)\right|_{a=0} \propto Q_{0}(\Gamma) .
\end{aligned}
$$

In other words, as $a$ varies from 0 to 1 , the states $f(\Gamma, a)$ follow the solution $f(\Gamma, t)$ as $t$ varies from 0 to $\infty$. Since the entropy increases with time for the solution $f(\Gamma, t)$, the function $S_{B}(a)=S_{B}(f(a))$ is a monotonically increasing function of the variable a. This condition, as well as the conditions (12), should be met by any method of 
constructing an approximation to the phase trajectory $f(\Gamma, a)$ (see conditions $1-6$ in the Introduction).

The simplest approximation based on the function $f^{*}$ of the preceeding section can be constructed as follows:

$$
f(\Gamma, a)= \begin{cases}(1-2 a) f_{0}(\Gamma)+2 a f^{*}(\Gamma) & \text { for } 0 \leqslant a \leqslant \frac{1}{2}, \\ 2(1-a) f^{*}(\Gamma)+(2 a-1) f^{0}(\Gamma) & \text { for } \frac{1}{2} \leqslant a \leqslant 1\end{cases}
$$

This approximation amounts to the two-step presentation of the relaxation from the initial state $f_{0}$ to the equilibrium state $f^{0}$ through the intermediate state $f^{*}$ of (2). The first step (parameter $a$ increases from 0 to $\frac{1}{2}$ ) is the relaxation directed along $Q_{0}$ up to the state $f^{*}$ in (2). The second step (parameter $a$ increases from $\frac{1}{2}$ to 1 ) is a linear relaxation from $f^{*}$ towards the equilibrium state. The last step can be viewed as the phase trajectory of a solution to the equation

$$
\partial_{t} f(\Gamma, t)=-k_{2}\left(f(\Gamma, t)-f^{0}(\Gamma)\right),
$$

with the initial condition $f^{*}$ in (2). In kinetic theory, an equation of the form (14) is known as the BGK-model [15]. The entropy increase along the second step is due to the well-known properties of Eq. (14).

Expression (13) demonstrates the advantage of using the state $f^{*}$ for the purpose of approximating the phase trajectory: conditions (12) are obviously satisfied, and also we have no worry about the entropy increase. Thus, all conditions $1-6$ mentioned in the Introduction are satisfied by the approximation (13) due to the features of the state $f^{*}$. To get explicit expressions, the estimate (9) can be used with no violations.

A disadvantage of the two-step approximation (13) is its non-smoothness at $a=\frac{1}{2}$. This can be improved as follows: Let us consider a triangle $T$ formed by the three states, $f_{0}, f^{*}$, and $f^{0}$, i.e. a closed set of convex linear combinations of these functions ${ }^{3}$. This object allows for a geometrical language to be used. A simple conjecture of the properties of the state $f^{*}$ is that all the elements of the triangle $T$ are nonnegative functions, and if $f$ belongs to $T$ then $C_{i}(f)=C_{i}\left(f_{0}\right)$, where $i=1, \ldots, k$. Therefore, a better approximation to the phase trajectory can be constructed as a smooth curve inscribed into the triangle $T$ in such a way that:

1. it begins in the state $f_{0}$ at $a=0$;

2. it is tangent to the side $L_{f_{0} f^{*}}=\left\{f \mid f=a_{1} f_{0}+a_{2} f^{*}, \quad a_{1} \geqslant 0, a_{2} \geqslant 0\right.$, $\left.0 \leqslant a_{1}+a_{2} \leqslant 1\right\}$ in the state $f_{0}$;

3. it ends in the equilibrium state $f^{0}$ at $a=1$.

Notice that the approximation (13) corresponds to the path from $f_{0}$ to $f^{0}$ over the two sides of the triangle $T$ : firstly, over the segment between $f_{0}$ and $f^{*}$, and, secondly, over the segment between $f^{*}$ and $f^{0}$.

The simplest form of such a smooth curve reads

$$
f_{g}(\Gamma, a)=f^{0}+\left(1-a^{2}\right)\left\{a g\left(f^{*}-f_{0}\right)+f_{0}-f^{0}\right\},
$$

${ }^{3}$ The state $f$ belongs to $T$ if $f=a_{1} f_{0}+a_{2} f^{*}+a_{3} f^{0}$, where $a_{i} \geqslant 0$ and $0 \leqslant a_{1}+a_{2}+a_{3} \leqslant 1$. 
where $g, 0<g \leqslant 1$, is an unknown parameter to be determined in a way that the entropy $S_{B}(a)$, calculated in the states (15), is an increasing function of $a$. An explicit sufficient method to estimate the value of parameter $g$ in (15) is developed in Appendix B.

Lastly, let us consider briefly a question of the time dependence of the approximations $f(\Gamma, a)$. Clearly, this question is relevant as soon as one looks for the approximate phase trajectories directly, rather than integrating Eq. (11) with respect to time. ${ }^{4}$ An answer assumes a dependence $a(t)$ and requires an ordinary differential equation for $a$. Such an equation should be obtained upon substitution of the expression for $f(\Gamma, a)$ into the originating kinetic equation (11) and by a further projection operation. More specifically, the equation for $a(t)$ has a form

$$
\frac{d a}{d t} \cdot \int \varphi(\Gamma, a) \frac{\partial f(\Gamma, a)}{\partial a} d \Gamma=\int \varphi(\Gamma, a) Q(f(\Gamma, a)) d \Gamma,
$$

where integration with the function $\varphi(\Gamma, a)$ establishes a projection operation. Usually, this is achieved by some moment projecting ( $\varphi$ is independent of $a$ ), but this choice is rather arbitrary. Another possibility is to choose the so-called thermodynamic projector [16]. Then Eq. (16) becomes the entropy balance equation along the path (15):

$$
\frac{d a}{d t} \cdot \frac{d S_{B}(a)}{d a}=\sigma_{B}(a)
$$

where $S_{B}(a)=-\int f(\Gamma, a) \ln f(\Gamma, a) d \Gamma$ and $\sigma_{B}(a)=-\int Q(f(\Gamma, a)) \ln f(\Gamma, a) d \Gamma$ are the entropy and the entropy production in the states $f(\Gamma, a)$ (Eq. (15)), respectively.

A further consideration of Eq. (17) is beyond the scope of this paper. Nevertheless, let us consider an asymptotic of the dynamics (17) for the motion from $f_{0}$ towards $f^{*}$. As above, we take $f(\Gamma, a)=(1-a) f_{0}+a f^{*}$. Eq. (17) for this function gives

$$
\begin{aligned}
& a(t) \sim \frac{1}{a^{*}} t, \quad a \ll 1, \\
& a(t) \sim \frac{\sigma_{B}^{*}}{\left(a^{*}\right)^{2} K_{0}} \sqrt{t}, \quad 1-a \ll 1,
\end{aligned}
$$

where $\sigma_{B}^{*}$ is the entropy production in the state $f^{*}$ and $K_{0}=\int\left(Q_{0}^{2} / f_{0}\right) d \Gamma$. A slowing down at a final stage is due to the fact that $d S_{B}(a) / d a \rightarrow 0$ as $a \rightarrow 1$, and $\sigma_{B}^{*}>0$.

\section{Relaxation of the Boltzmann gas}

The direct and the simplest application of the approach is the space-independent Boltzmann equation. In what follows, $\Gamma$ is the velocity $\mathbf{v}$ and $f(\Gamma)$ is the one-body

\footnotetext{
${ }^{4}$ This question is typical of many different approximations used in the kinetic theory, cf. [12, 16].
} 
distribution function $f(\mathbf{v})$ which obeys the equation:

$$
\partial_{t} f(\mathbf{v}, t)=Q(f)
$$

with $Q(f)$ being the Boltzmann collision integral.

As the first example we consider the following form of the collision integral $Q$ :

$$
Q(f)=\int d \mathbf{w} \int d \hat{\mathbf{n}} \gamma(\hat{\mathbf{g}} \cdot \hat{\mathbf{n}})\left\{f\left(\mathbf{v}^{\prime}, t\right) f\left(\mathbf{w}^{\prime}, t\right)-f(\mathbf{v}, t) f(\mathbf{w}, t)\right\},
$$

where the function $\gamma$ depends solely on a scalar product of unit vectors $\hat{\mathbf{g}}=(\mathbf{v}-\mathbf{w})$ / $|\mathbf{v}-\mathbf{w}|$ and $\hat{\mathbf{n}}=\left(\mathbf{v}^{\prime}-\mathbf{w}^{\prime}\right) /|\mathbf{v}-\mathbf{w}|$, while $\mathbf{v}^{\prime}=\frac{1}{2}(\mathbf{v}+\mathbf{w}+\hat{\mathbf{n}}|\mathbf{v}-\mathbf{w}|)$, and $\mathbf{w}^{\prime}=\frac{1}{2}(\mathbf{v}+\mathbf{w}-$ $\hat{\mathbf{n}}|\mathbf{v}-\mathbf{w}|$ ) (notations are standard and follow [9]). The Boltzmann equation (18), with the collision integral (19), corresponds to the model repelling potential proportional to the inverse fourth degree of distance (3D Maxwellian molecules, see e.g. [8]). The reason to consider this model here is that it has an exact solution, the famous BKWmode discovered by Bobylev [2] and Krook and $\mathrm{Wu}$ [3]. The BKW-mode represents the following one-parametric set of the distribution functions $f(c, \mathbf{v})$ :

$$
f(c, \mathbf{v})=\frac{1}{2}\left(\frac{2 \pi}{c}\right)^{-3 / 2} \exp \left\{-\frac{c v^{2}}{2}\right\}\left((5-3 c)+c(c-1) v^{2}\right),
$$

where the parameter $c$ spans the segment $\left[1, \frac{5}{3}[\right.$; value $c=1$ corresponds to the final Maxwell distribution $f^{0}(\mathbf{v})=f(1, \mathbf{v})=(2 \pi)^{-3 / 2} \exp \left\{-v^{2} / 2\right\}$. As $c$ decays from a given value $c_{0}$, where $1<c_{0}<\frac{5}{3}$, to the value $c=1$, the functions $f(c, \mathbf{v})$ of Eq. (20) describe the phasetrajectory of the BKW-mode (time dependence of $c$ is unimportant in the present context, see e.g. [9]).

Considering the states (20) as the initial states in the procedure described above, we can construct the upper limiting states, $f^{*}(c, \mathbf{v})=f(c, \mathbf{v})+a^{*}(c) Q(c, \mathbf{v})$, for each allowed value of $c$. Firstly, we compute the collision integral (19) in the states (20) to get the functions $Q(c, \mathbf{v})$ :

$$
Q(c, \mathbf{v})=\frac{\lambda}{2}(c-1)^{2}\left(\frac{2 \pi}{c}\right)^{-3 / 2} \exp \left\{-\frac{c v^{2}}{2}\right\}\left(15-10 c v^{2}+c^{2}\left(v^{2}\right)^{2}\right)
$$

where $\lambda$ is a constant: $\lambda=\frac{1}{8} \int d \hat{\mathbf{n}} \gamma(\hat{\mathbf{k}} \cdot \hat{\mathbf{n}})\left(1-(\hat{\mathbf{k}} \cdot \hat{\mathbf{n}})^{2}\right)$.

Expression (21) suggests a simple structure of the velocity space partition into the domains $V_{+}(c)$ and $V_{-}(c)$ (corresponding to the domains $\Gamma_{ \pm}$introduced in Section 2); namely, for a given $c$, the function $(21)$ is positive inside a sphere of radius $v_{-}(c)=$ $\sqrt{c^{-1}(5-\sqrt{10})}$ and outside a larger sphere of radius $v_{+}(c)=\sqrt{c^{-1}(5+\sqrt{10})}$ (both spheres are centered in $\mathbf{v}=0$ ), while it is negative inside a spheric layer between these spheres:

$$
\begin{aligned}
& V_{-}(c)=\left\{\mathbf{v}\left|v_{-}(c)<\right| \mathbf{v} \mid<v_{+}\right\} \\
& V_{+}(c)=\left\{\mathbf{v}\left|v_{-}(c)>\right| \mathbf{v} \mid\right\} \cup\left\{\mathbf{v}|| \mathbf{v} \mid>v_{+}\right\}
\end{aligned}
$$


The limiting states $f^{*}(c, \mathbf{v})$ are given by the following expression:

$$
\begin{aligned}
f^{*}(c, \mathbf{v})= & \frac{1}{2}\left(\frac{2 \pi}{c}\right)^{-3 / 2} \exp \left\{-\frac{c v^{2}}{2}\right\} \\
& \times\left(5-3 c+15 a^{*}(c)+\left(c-1-10 a^{*}(c)\right) c v^{2}+a^{*}(c) c^{2}\left(v^{2}\right)^{2}\right),
\end{aligned}
$$

where $a^{*}(c)$ is a solution of Eq. (4):

$$
\int Q(c, \mathbf{v}) \ln \left(f(c, \mathbf{v})+a^{*}(c) \frac{Q(c, \mathbf{v})}{\lambda(c-1)^{2}}\right) d \mathbf{v}=0 .
$$

Accounting for the partition (22), all the parameters (10) required for the explicit evaluation are expressed in terms of definite one-dimensional integrals. Thus, we obtain the first approximate $a_{1}^{*}(c)$. Numerical results are presented in the Table 1 (second column) for the three different values of the parameter $c$. In this special case we can compare $a_{1}^{*}(c)$ with $a_{\max }(c)$, for which the function $f(c, \mathbf{v})+a Q(c, \mathbf{v}) /\left[\lambda(c-1)^{2}\right]$ looses positivity (i.e. this function becomes negative for some $\mathbf{v}$, as $a>a_{\max }(c)$ ). A ratio $a_{1}^{*}(c) / a_{\max }(c)$ is given in the third column of Table 1 . A "step" in the direction $Q(c, \mathbf{v})$ which is allowed due to the entropy reasons is never negligible in comparison to that determined by the positivity reasons.

We can now use Eq. (23) to get the approximations of phase trajectories (13) and (15) as described in section 3 and in Appendix B. The derivation of the parameter $g$ in expression (15), in accordance with the procedure of Appendix B, Section B.5, gives $g=1$ for all initial states (20).

To make a comparison with the exact result (20), we have considered the dependences of the normalized moments $m_{k}\left(m_{l}\right)$ :

$$
m_{s}(f)=\frac{\int\left(v^{2}\right)^{s} f d \mathbf{v}}{\int\left(v^{2}\right)^{s} f^{0} d \mathbf{v}}, \quad s=0,1,2, \ldots
$$

Typical dependences of the higher moments $(k \geqslant 3)$ on the lowest non-trivial moment $(l=2)$ are presented in Fig. 1(a) and (b) for a considerably non-equilibrium initial state (20) with $c=1.42$.

An error of the approximation (15) was estimated as follows: In each moment plane $\left(m_{k}, m_{l}\right)$, the approximation (15) and the BKW-mode (20) generate two sets (the two curvilinear segments) $X_{k l}$ and $Y_{k l}$, respectively. Firstly, in order to eliminate the contribution from the difference in the total variation of the moments, we rescale

\begin{tabular}{l}
$\begin{array}{l}\text { Table } 1 \\
\text { The limiting states for the } \\
\text { Maxwellian molecules }\end{array}$ \\
\hline \begin{tabular}{lll} 
c & $a_{1}^{*}$ & $a_{1}^{*} / a_{\max }$ \\
\hline 1.12 & $3.1779 \times 10^{-3}$ & 0.2221 \\
1.24 & $1.1660 \times 10^{-2}$ & 0.4291 \\
1.48 & $3.8277 \times 10^{-2}$ & 0.7087 \\
\hline
\end{tabular}
\end{tabular}



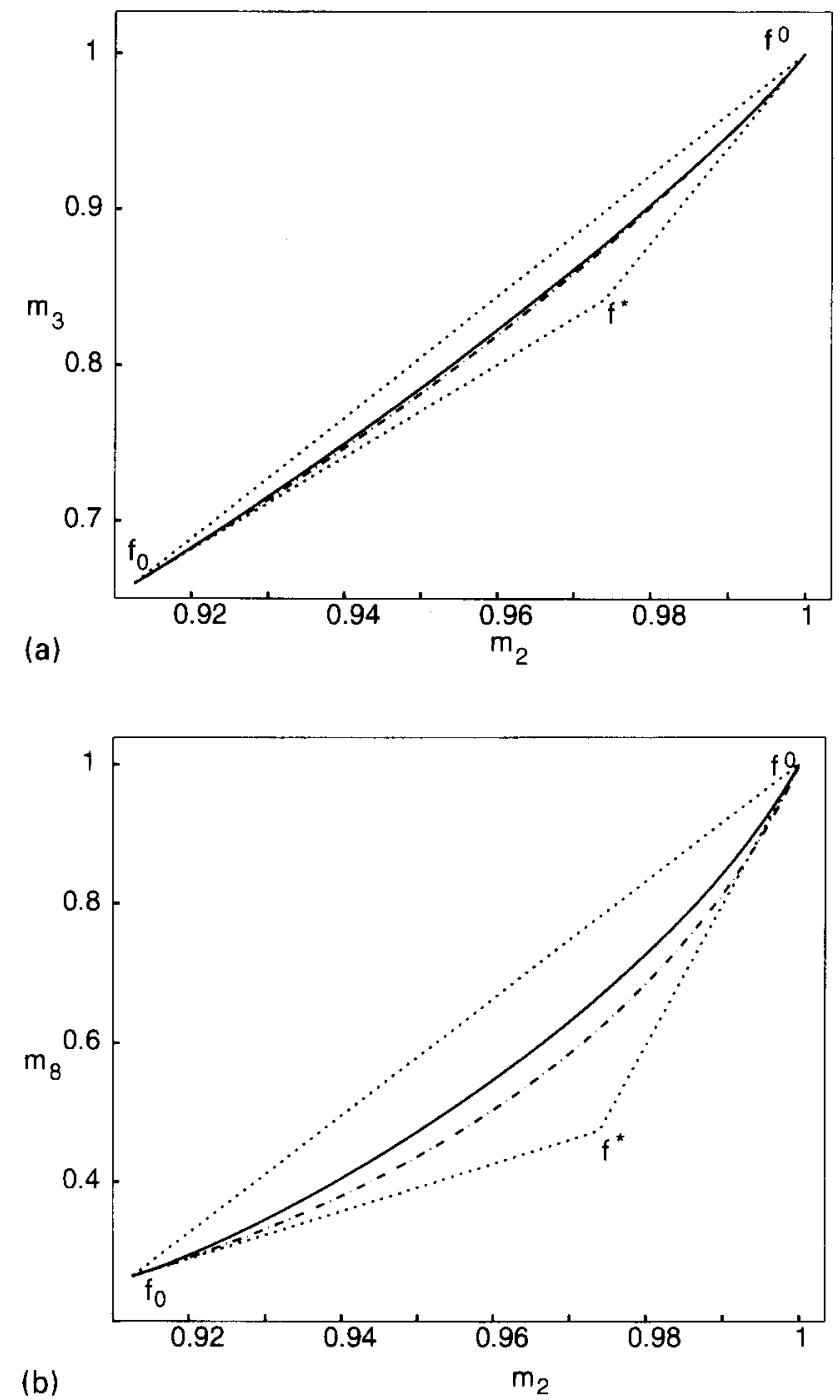

Fig. 1. Moment dependences for the Maxwellian molecules: (a) $m_{3}$ vs. $m_{2}$; (b) $m_{8}$ vs. $m_{2}$. The initial state $f_{0}$ is the function (20) with $c=1.42$; punctuated contour; the image of the triangle $T$; punctuated dashed line; the BKW-mode; solid line: the smooth approximation (15); punctuated path $f_{0} \rightarrow f^{*} \rightarrow f^{0}$; the non-smooth approximation (13).

the variables:

$$
\hat{m}_{i}=m_{i} / \Delta_{i}, \quad i=k, l,
$$

where

$$
\Delta_{i}=\max _{\mathbf{x}, \mathbf{x}^{\prime} \in X_{k l} \cup Y_{k i}}\left|x_{i}-x_{i}^{\prime}\right| .
$$


Secondly, in the plane $\left(\hat{m}_{k}, \hat{m}_{l}\right)$, we compute the Hausdorff distance $\mathrm{d}_{k l}$ between the two corresponding sets $\hat{X}_{k l}$ and $\hat{Y}_{k l}$ :

$$
\mathbf{d}_{k l}=\max \left\{\max _{\mathbf{x} \in \hat{X}_{k l}} \min _{\mathbf{y} \in \hat{Y}_{k l}} d(\mathbf{x}, \mathbf{y}), \max _{\mathbf{y} \in \hat{Y}_{k l}} \min _{\mathbf{x} \in \hat{X}_{k l}} d(\mathbf{x}, \mathbf{y})\right\},
$$

where $d(\mathbf{x}, \mathbf{y})$ is the standard Euclidian distance between the two points. Finally, the error $\delta_{k l}$ was estimated as the normalized distance $d_{k l}$ :

$$
\delta_{k l}=\frac{d_{k l}}{D_{k l}} \cdot 100 \%,
$$

where

$$
D_{k l}=\max _{\mathbf{x}, \mathbf{y} \in \hat{Y}_{k l} \cup \hat{X}_{k l}} d(\mathbf{x}, \mathbf{y}) .
$$

The error $\delta_{k 2}$ of the plots in Figs. 1(a) and (b) is presented in Table 2 for several values of the parameter $c$.

The quality of the smooth approximation (15) is either good or reasonable up to $k \sim 10$, depending on the closeness of the initial state to the equilibrium state, and it becomes worse when either $k$ increases, or the initial state is taken very far from the equilibria (i.e. when $c$ is close to $\frac{5}{3}$ ). For the moments of a very high order, the approximation with the smooth function (15) is only qualitative. On the other hand, the two-step (non-smooth) approximation (13) provides a much better approximation for very high moments ( $k \sim 40$ and higher). The explanation is as follows: as is well known, the BKW-mode (20) demonstrates a very rapid relaxation of higher moments to their equilibrium values. Therefore, as it might be expected, the relaxation in the direction $Q_{0}$ brings us to the state where the higher moments are practically the same as in the equilibrium.

Table 2

The error $\delta_{k 2}$ (27) of the approximation (15) for the Maxwellian molecules with the initial data (20)

\begin{tabular}{llllll}
\hline$k$ & $c=1.12$ & $c=1.24$ & $c=1.36$ & $c=1.48$ & $c=1.59$ \\
\hline 3 & 0.31 & 0.33 & 0.30 & 0.41 & 0.70 \\
4 & 0.44 & 0.47 & 0.44 & 0.81 & 1.57 \\
5 & 0.58 & 0.55 & 0.71 & 1.41 & 2.67 \\
6 & 0.71 & 0.57 & 1.10 & 2.20 & 3.97 \\
7 & 0.81 & 0.62 & 1.58 & 3.14 & 5.40 \\
8 & 0.89 & 0.84 & 2.19 & 4.19 & 6.93 \\
9 & 0.95 & 1.11 & 2.87 & 5.34 & 8.52 \\
10 & 0.99 & 1.41 & 3.64 & 6.55 & 10.11 \\
20 & 1.46 & 6.77 & 12.91 & 18.15 & 22.87 \\
50 & 10.38 & 28.47 & 27.36 & 30.81 & 33.94 \\
100 & 21.76 & 29.26 & 32.49 & 34.78 & 37.22 \\
\hline & & & & &
\end{tabular}


The second test example is the VHP model $[4,5]$. The distribution function $F(x)$ depends on the phase variable $x$, where $0 \leqslant x \leqslant \infty$, and is governed by the following kinetic equation:

$$
\partial_{t} F(x, t)=\int_{x}^{\infty} d u \int_{0}^{u} d y[F(y, t) F(u-y, t)-F(x, t) F(u-x, t)] .
$$

This model implies the two conservation laws:

$$
\begin{aligned}
& N=\int_{0}^{\infty} F(x, t) d x=1, \\
& E=\int_{0}^{\infty} x F(x, t) d x=1,
\end{aligned}
$$

and has the entropy $S_{B}(F)=-\int_{0}^{\infty} F(x) \ln F(x) d x$. The equilibrium distribution reads $F^{0}(x)=\exp (-x)$. The general solution to this model is known $[4,5,9]$.

The first set of initial conditions which was tested is as follows:

$$
F_{0}(x, \beta)=\beta((2-\beta)+\beta(\beta-1) x) \exp (-\beta x),
$$

where $1 \leqslant \beta<2$, the value $\beta=1$ corresponds to the equilibrium state $F_{0}(x, 1)=F^{0}(x)$.

In accordance with $[4,5,9]$, the exact solution to Eq. (28) with the initial data (29) is as follows:

$$
\begin{aligned}
& F_{e x}(x, \beta, t)=\frac{A z_{+}+C}{z_{+}-z_{-}} \mathrm{e}^{x z_{+}}+\frac{A z_{-}+C}{z_{-}-z_{+}} \mathrm{e}^{x z_{-}}, \\
& z_{ \pm}=-\frac{t+2 \beta}{2} \pm \sqrt{\left(\frac{t+2 \beta}{2}\right)^{2}-C} \\
& A=1-(\beta-1)^{2} \mathrm{e}^{-t} ; \quad C=t+2 \beta-1+\mathrm{e}^{-t}(\beta-1)^{2} .
\end{aligned}
$$

A comparison of the smooth approximation (15) with the exact solution (30) demonstrates the same quality as for the example of the Maxwellian molecules. As above, the normalized moments $m_{k}$ were compared, where

$$
m_{k}=\frac{\int_{0}^{\infty} x^{k} F(x) d x}{\int_{0}^{\infty} x^{k} F^{0}(x) d x} .
$$

In Table 3, the error $\delta_{k 2}$ given in Eq. (27) is represented for several values of the parameter $\beta$, while Fig. 2 illustrates the typical moment behavior. We also represent in this figure a result of a correction to the approximation (15) due to the first iteration of a method introduced in [11] (see additional explanations in the next section).

The second set of the initial conditions for the VHP model (28) was considered as follows:

$$
F_{0}(x, \lambda)=\exp (-2 x)\left\{1+\frac{1}{2} \lambda+2 x^{2}(1-\lambda)+\frac{1}{3} \lambda x^{4}\right\}
$$


Table 3

The Error $\delta_{k 2}(27)$ of the approximation (15) for the VHP model with the initial data (29)

\begin{tabular}{lllllll}
\hline$\beta$ & $k$ & \multicolumn{1}{l}{$k$} \\
\cline { 2 - 7 } & 4 & 6 & 8 & 10 & 20 & 100 \\
\hline 1.2 & 0.95 & 1.81 & 2.26 & 2.23 & 2.24 & 9.64 \\
1.6 & 0.88 & 1.89 & 2.59 & 2.77 & 6.14 & 24.29 \\
1.9 & 1.16 & 1.65 & 1.45 & 3.16 & 12.7 & 28.22 \\
\hline
\end{tabular}

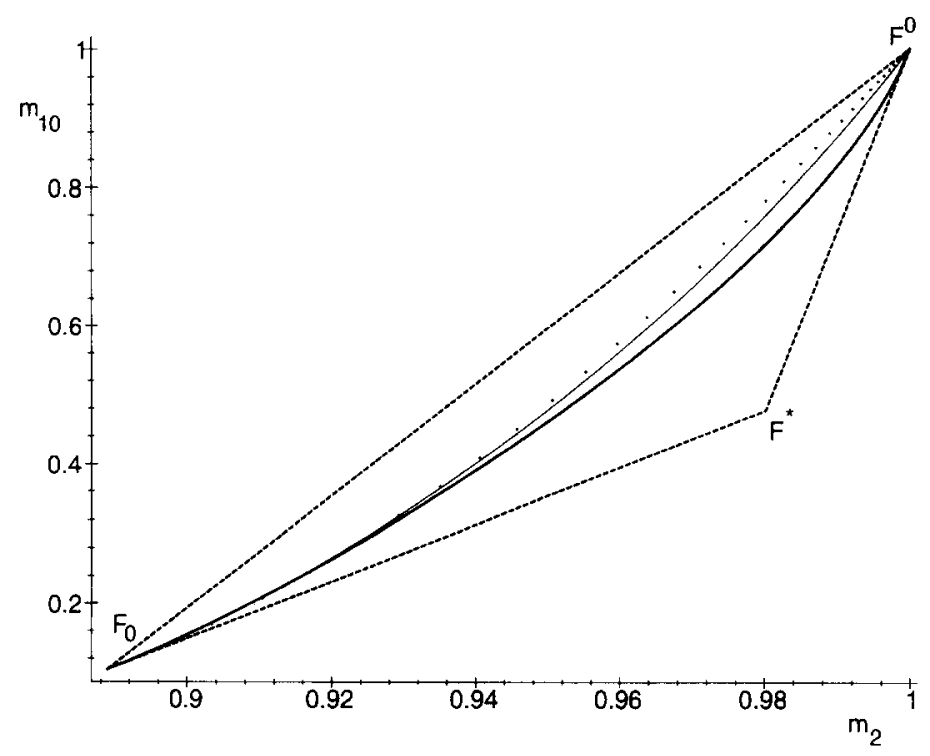

Fig. 2. Moment dependence $m_{10}$ vs. $m_{2}$ for the VHP model with the initial condition (29), $\beta=1.5$ : dots: the exact solution (30); bold line: the smooth approximation (15); solid line: the first correction to the approximation (15).

where $0<\lambda<\frac{1}{5}(7+\sqrt{19})$. The exact solution to Eq. (28) with the initial condition (31) has been considered in [5]. This solution demonstrates the so-called Tjon overshoot effect [17]. Recall that the Tjon effect occurs when the distribution function becomes overpopulated for some velocities in comparison to both the initial and the equilibrium states. This effect was studied intensively for the soluble Boltzmann-like kinetic equations, like the Maxwellian molecules (19), the VHP model (28), and others (see $[9,18]$ and references therein; it is worthwhile to mention here extensive studies of the Tjon-like effects in chemical kinetics $[19,14])$.

The approximation (15) for the VHP model (28) with the initial condition (31) also demonstrates the overshoot just mentioned. In the moment representation as above, the overshoot of the moments is clearly seen in Figs. 3(a) and (b). The quality 

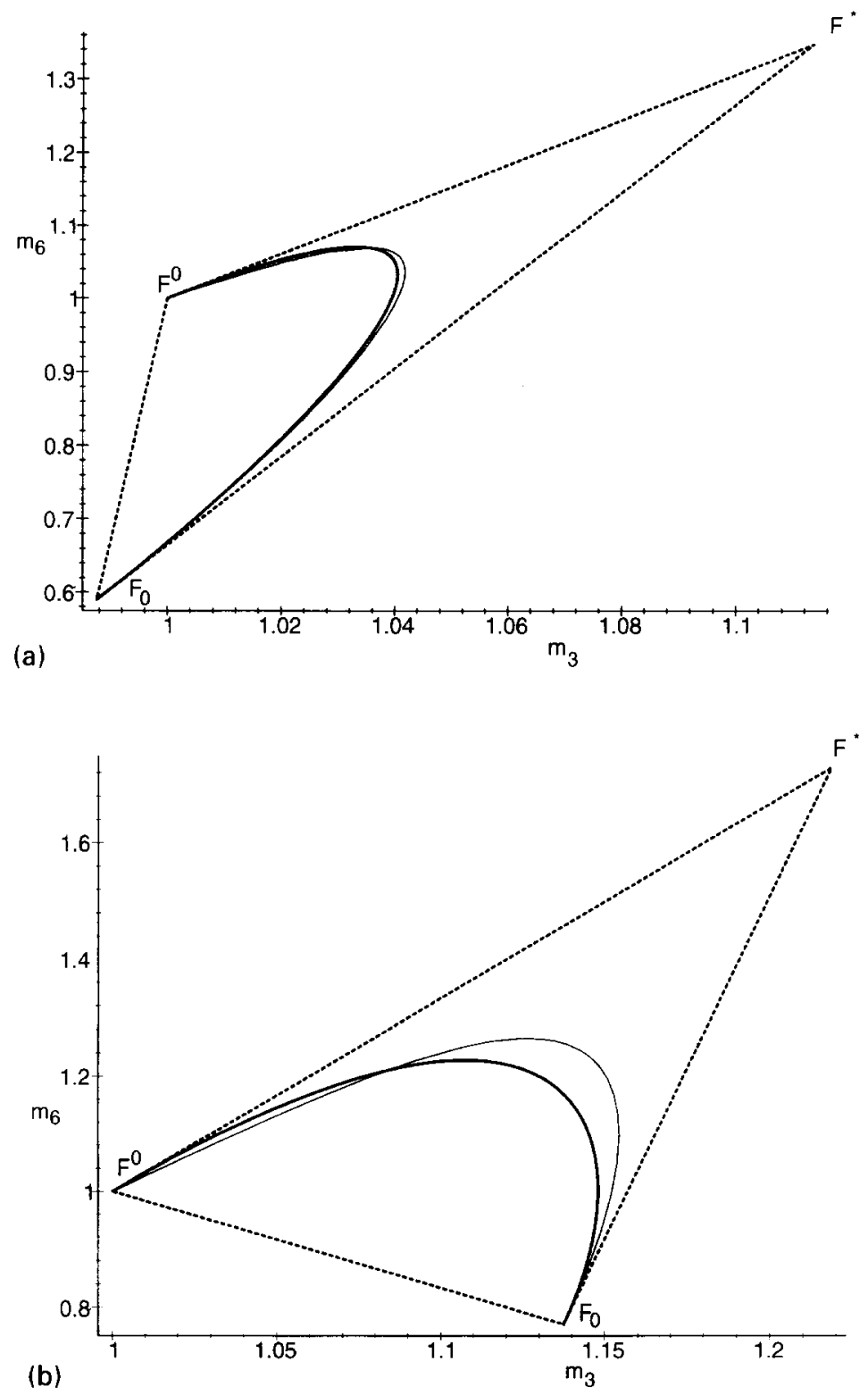

Fig. 3. Moment dependences $m_{6}$ vs. $m_{3}$ for the VHP model with the initial condition: (31) (a) $\lambda=0.6$; (b) $\lambda=0.9$; solid line: the exact solution [5]; bold line: the smooth approximation (15).

of the approximation with respect to the error (27) is the same as in the examples above.

Our final example is the Boltzmann equation (18) for the hard spheres to demonstrate that the computations are equally possible for models where exact solutions are not available. Figs. 4(a) and (b) demonstrate the moment dependences, as they appear in the approximation (15), when the initial state is taken as in Fig. 1, i.e. again on the 

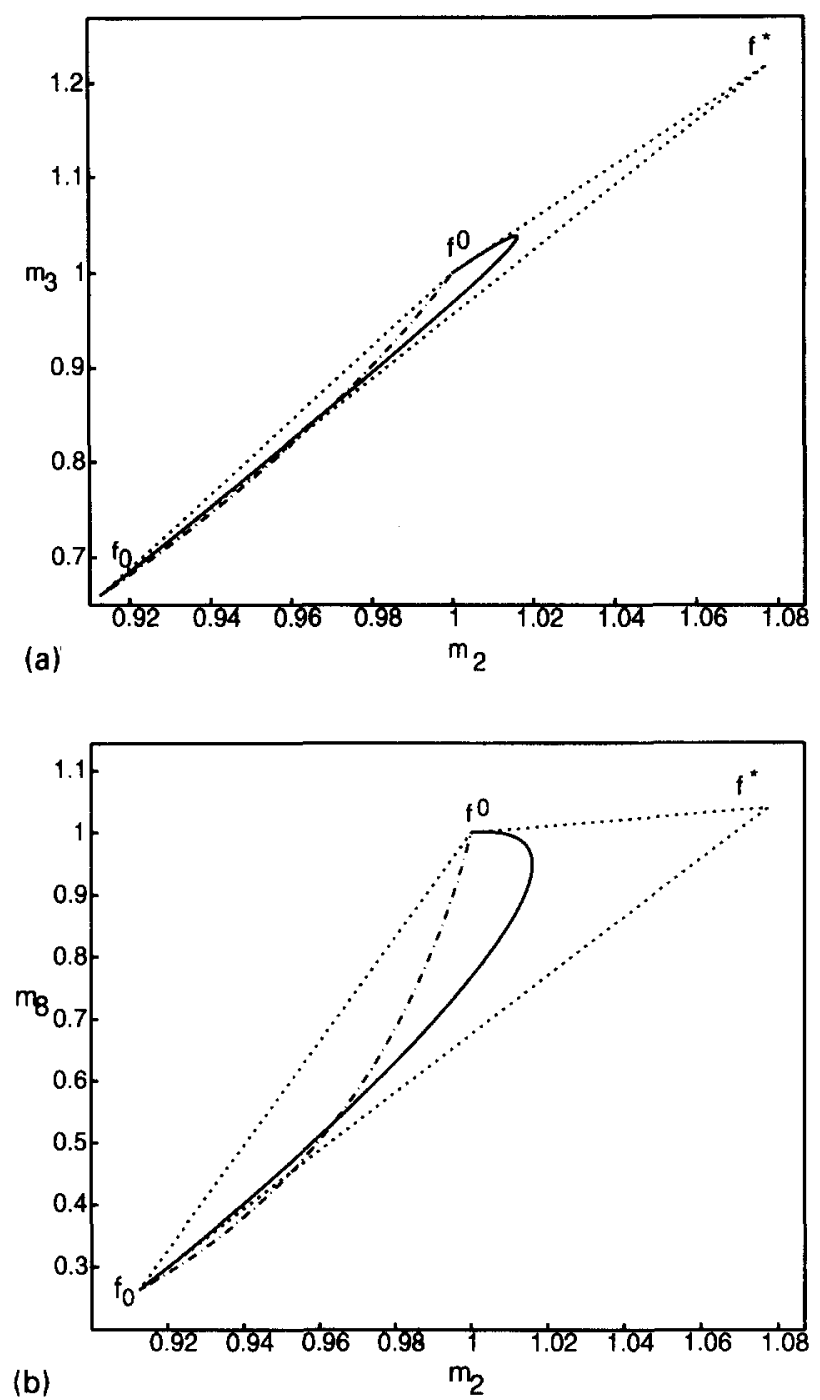

Fig. 4. Moment dependences for the hard spheres: (a) $m_{3}$ vs. $m_{2}$; (b) $m_{8}$ vs. $m_{2}$. The initial state $f_{0}$ is the function (20) with $c=1.42$; punctuated dashed line: the BKW-mode for the Maxwellian molecules; solid line: the smooth approximation (15).

BKW set (20). The moment dependence of the BKW-mode is also represented in these figures. 5

\section{Discussion}

The primary results of the paper are the following.

${ }^{5}$ Of course, the BKW-mode itself is neither exact nor the approximate solution for the hard spheres. 
1. The description of the $Q_{0}$-dominated kinetics and of its equilibrium state $f^{*}$. The state $f^{*}$ is explicitly evaluated.

2. The explicit construction of the approximate phase trajectory $f(\Gamma, a)$ for (nonlinear) space-independent kinetic equations.

The approach used can be called "geometrical" since it avoids an explicit integration of kinetic equations in time. In point 1 , it stays at variance with many alternative approaches to the early-time evolution, which usually involve the time integration over the first few collisions. These methods encounter two general difficulties: the time of integration cannot be defined precisely, and approximations involved can violate the entropy increase and the positivity. These difficulties are avoided in the present approach. On the other hand, the presentation of the $Q_{0}$-dominated relaxation is itself an ansatz, whose relevance to the actual process can be judged only a posteriori. As the examples show, it appears to be reasonable indeed to speak about such a dynamics. It is remarkable that the limiting state is at a significant variance with both the initial and the equilibrium states. In other words, irrespective of how short in time the initial stage of the relaxation might be, a change of the state can be big.

Concerning point 2 , it is worthwhile to notice that, though the space-independent problem is too "refined", it nevertheless gives a good example of a problem without small parameters. It is rather remarkable that the global requirements to the trajectory (e.g. the entropy increase) are accomplished with the direct local analysis (Appendix B). Estimations in this part are sufficient and can be enhanced.

Final comments concern a further treatment of the space-independent relaxation. As it was already mentioned at the end of the Introduction, the goal now is to develop a procedure of corrections to the approximate phase trajectory. In other words, what we need is a sequence of the functions $f_{0}(\Gamma, a), f_{1}(\Gamma, a), \ldots$, which converges to the exact trajectory, and where $f_{0}(\Gamma, a)$ is the initial (global) approximation to the trajectory. Again, a general obstacle is the absence of a small parameter in the problem. However, a recently developed method [12] appears to be appropriate (at least formally) since it is based on the Newton method and not on the small parameter expansions. It turns out that smoothness and all the requirements listed in the Introduction should be met by any initial approximation $f_{0}(\Gamma, a)$ chosen for this procedure. Thus, the approximation (15) can be used for this purpose. We have already advanced this method with a result of the first Newton correction to the approximation (15) for the VHP model (see Fig. 2). However, a further consideration will take us far afield, and we defer a detailed presentation of the correction procedure for a further publication.

\section{Acknowledgements}

We acknowledge the useful comments of Dr. G. Dukek and are grateful to the referee for a suggestion to take the VHP model for a test of the method; one of the authors (IVK) is grateful to Prof. A. V. Bobylev and to Prof. H. Neunzert for a discussion of the results, and thanks the Alexander von Humboldt Foundation for a possibility of a 
research stay at the University of Ulm; this work was supported in part by RFFI grant No. 95-02-03836-a (ANG and IVK).

\section{Appendix A. Evaluation of limiting state}

Let us introduce a normalization of the partition $Q_{0}^{ \pm}(\Gamma)$ :

$$
q_{0}^{ \pm}(\Gamma)=q^{-1} Q_{0}^{ \pm}(\Gamma), \quad q=\int_{\Gamma_{ \pm}} Q_{0}^{ \pm}(\Gamma) d \Gamma .
$$

Introducing a variable $b=q a$, so that $f^{*}=f_{0}(\Gamma)+b^{*} q_{0}(\Gamma)$, where $q_{0}(\Gamma)=q^{-1} Q_{0}(\Gamma)$, Eq. (4) can be rewritten as follows:

$$
A_{+}\left(b^{*}\right)=A_{-}\left(b^{*}\right),
$$

where

$$
A_{ \pm}(b)=\int_{\Gamma_{ \pm}} q_{0}^{ \pm}(\Gamma) \ln \left(f_{0}(\Gamma) \pm b q_{0}^{ \pm}(\Gamma)\right) d \Gamma .
$$

It is easy to check the following properties of the functions $A_{ \pm}$of (A.3):

1. A domain of $A_{+}$is $] b_{+},+\infty$ [, where $b_{+}<0$, and a domain of $A_{-}$is ] $-\infty, b_{-}$, where $b_{-}>0$. The functions $A_{ \pm}$have logarithmic singularities at the points $b_{ \pm}$, respectively.

2. The functions $A_{ \pm}$are monotonic and concave inside their domains.

3. An inequality holds as: $A_{+}(0)-A_{-}(0)=-q^{-1} \sigma_{0}<0$, where $\sigma_{0}=-\int Q_{0}(\Gamma) \ln$ $f_{0}(\Gamma) d \Gamma$ is the entropy production in the state $f_{0}$.

One has to solve Eq. (A.2) to get the approximations $b_{1}^{*}, b_{2}^{*}, \ldots$ not greater than the unknown exact value $b^{*}$. To get a relevant estimate of $b^{*}$, it is convenient to make use of concavity properties of the functions (A.3). Indeed, for positive $b$, the function $A_{-}$is estimated from below as:

$$
A_{-}(b) \geqslant A_{-}(0)+\ln \left(1-\alpha_{1} b\right) \text {. }
$$

Here $\alpha_{1}$ is the inverse of $b_{-}$:

$$
x_{1}=\sup _{\Gamma \in \Gamma_{-}} \frac{q_{0}^{-}(\Gamma)}{f_{0}(\Gamma)}=\sup _{\Gamma \in \Gamma_{-}} \frac{Q_{0}^{-}(\Gamma)}{q f_{0}(\Gamma)}=q^{-1} \alpha,
$$

while $\alpha$ was introduced in (10).

On the contrary, the function $A_{+}$should be estimated from above. Noting that the function $\exp A_{+}$is also monotonic and concave, we can write for positive $b$

$$
A_{+}(b) \leqslant A_{+}(0)+\ln \left(1+b \frac{d A_{+}(0)}{d b}\right),
$$


where

$$
\frac{d A_{+}(0)}{d b}=\int_{\Gamma_{+}} \frac{\left(q_{0}^{+}(\Gamma)\right)^{2}}{f_{0}(\Gamma)} d \Gamma=q^{-1} \beta,
$$

and $\beta$ was introduced in (10).

Equating the RHS of Eq. (A.5) to the RHS of Eq. (A.4), and solving a linear equation obtained, we get an estimate $b_{1}^{*} \leqslant b^{*}$. Next, turning back to the variable $a$, we get the estimation $a_{1}^{*}(9)$ and (10). One can readily recognize that the procedure just described is the first iterate of the Newton method for Eq. (A.2) (slightly modified by making use of the concavity to guarantee $\left.a_{1}^{*} \leqslant a^{*}\right)$. Further iterations are performed in the same manner.

\section{Appendix B. Smooth approximations of the phase trajectories}

\section{B.1. The triangle of model motions}

Notation $\overline{\operatorname{conv}}\left\{f_{1}, \ldots, f_{k}\right\}$ will stand for a closed convex linear envelope of the functions $f_{1}, \ldots, f_{k}$, and we drop the variable $\Gamma$. In particular, the triangle $T$ introduced in Section 3 reads

$$
T=\overline{\operatorname{conv}}\left\{f_{0}, f^{*}, f^{0}\right\} .
$$

A function from the triangle $T$ can be specified with two parameters $\xi$ and $\eta$ as $f(\xi, \eta)$ :

$$
f(\xi, \eta)=f^{0}+\xi\left\{\eta\left(f^{*}-f_{0}\right)+f_{0}-f^{0}\right\}, \quad 0 \leqslant \xi, \eta \leqslant 1 .
$$

A shift of the function $f(\xi, \eta)$ under a variation of $\xi$ and of $\eta$ reads

$$
\begin{aligned}
\Delta f(\xi, \eta) & =\partial_{\xi} f(\xi, \eta) \Delta \xi+\partial_{\eta} f(\xi, \eta) \Delta \eta+o(\Delta \xi, \Delta \eta) \\
& =\left(f(\xi, \eta)-f^{0}\right) \xi^{-1} \Delta \xi+a^{*} Q_{0} \xi \Delta \eta+o(\Delta \xi, \Delta \eta)
\end{aligned}
$$

This shift is a superposition of two shifts: a shift towards $f^{0}$ and a shift in the direction $Q_{0}$. We further refer to these as the BGK-motion and the $Q_{0}$-motion, respectively. The differential of the entropy $S_{B}(\xi, \eta)=S_{B}(f(\xi, \eta))$ is

$$
d S_{B}(\xi, \eta)=-\sigma_{1}(\xi, \eta) \xi^{-1} d \xi+\sigma_{2}(\xi, \eta) \xi d \eta
$$

where

$$
\begin{aligned}
& \sigma_{1}(\xi, \eta)=\int\left(f(\xi, \eta)-f^{0}\right) \ln \frac{f(\xi, \eta)}{f^{0}} d \Gamma, \\
& \sigma_{2}(\xi, \eta)=\int\left(f_{0}-f^{*}\right) \ln f(\xi, \eta) d \Gamma=-a^{*} \int Q_{0} \ln f(\xi, \eta) d \Gamma,
\end{aligned}
$$

are the entropy production in the BGK-motion and in the $Q_{0}$-motion, respectively. 
Introducing smooth dependences, $\xi(a)$ and $\eta(a)$ where $0 \leqslant a \leqslant 1$, and requiring

$$
\begin{aligned}
& 0 \leqslant \xi(a), \eta(a) \leqslant 1, \quad \xi(0)=1, \quad \xi(1)=0, \\
& \eta(0)=0, \quad \eta(1)<\infty, \\
& \left.\frac{d \xi(a)}{d a}\right|_{a=0}=0,\left.\quad \frac{d \eta(a)}{d a}\right|_{a=0}=\gamma, \quad 0<\gamma \leqslant 1,
\end{aligned}
$$

we obtain a one-parametric set $f(a)=f(\xi(a), \eta(a))$. Geometrically, $f(a)$ is a smooth curve located in $T$. This curve starts in $f_{0}$ at $a=0$, ends in $f^{0}$ at $a=1$, and is tangent to the side of $T, L_{f_{0} f^{*}}=\overline{\operatorname{conv}}\left\{f_{0}, f^{*}\right\}$, at $a=0$. Further, only monotonic functions $\xi(a)$ and $\eta(a)$ will be considered:

$$
\frac{d \xi(a)}{d a} \leqslant 0, \quad \frac{d \eta(a)}{d a} \geqslant 0 .
$$

The crucial point is that the function $f(a)$ should have a correct entropy behavior. More specifically, we require that the entropy $S_{B}(a)=S_{B}(f(a))=S_{B}(f(\xi(a), \eta(a)))$ is a monotonic function:

$$
\frac{d S_{B}(a)}{d a}=-\sigma_{1}(\xi(a), \eta(a)) \xi^{-1}(a) \frac{d \xi(a)}{d a}+\sigma_{2}(\xi(a), \eta(a)) \xi(a) \frac{d \eta(a)}{d a} \geqslant 0 .
$$

Since $\sigma_{1}(\xi, \eta)$ is non-negative everywhere in $T$, a sufficient condition for inequality (B.7) to be valid for any pair of functions $\xi(a)$ and $\eta(a)$ with the properties (B.5) and (B.6) is that $\sigma_{2}(\xi, \eta)$ is non-negative everywhere in $T$. However, this situation might not be realized for arbitrary $f_{0}$ and $Q_{0}$. For a general situation, we execute the following procedure:

1. We derive a subset of $T$ inside which $\sigma_{2}$ is non-negative. This subset includes $f_{0}$, and will be constructed as a triangle $T^{\prime} \subseteq T$.

2. We adjust the functions $\xi(a)$ and $\eta(a)$ in such a way that $\sigma_{1}(a)$ dominates $\sigma_{2}(a)$ outside $T^{\prime}$.

\section{B.2. The triangle $T^{\prime}$}

Let us introduce a different specification of the functions in the triangle $T$. Denote

$$
f_{\mathrm{I}}(y)=(1-y) f_{0}+y f^{*}, \quad f_{2}(y)=(1-y) f_{0}+y f^{0}, \quad 0 \leqslant y \leqslant 1 .
$$

The functions in $T$ are labeled with two parameters $x$ and $y$ :

$$
f(x, y)=(1-x) f_{1}(y)+x f_{2}(y), \quad 0 \leqslant x, y \leqslant 1
$$

Let us derive $y^{\prime}$, where $0<y^{\prime} \leqslant 1$, in such a way that $\sigma_{2}$ is non-negative everywhere in the triangle $T^{\prime}=\overline{\operatorname{conv}}\left\{f_{0}, f_{1}\left(y^{\prime}\right), f_{2}\left(y^{\prime}\right)\right\}$. 
Introducing a representation $\sigma_{2}(x, y)=\sigma_{2}^{+}(x, y)-\sigma_{2}^{-}(x, y)$, where

$$
\sigma_{2}^{+}(x, y)=\int f_{0} \ln f(x, y) d \Gamma, \quad \sigma_{2}^{-}(x, y)=\int f^{*} \ln f(x, y) d \Gamma,
$$

we notice that the functions $\sigma_{2}^{ \pm}(x, y)$ are concave in the variable $y$ on the segment $[0,1]$, for any fixed $x$. Now we apply the standard estimations of a smooth concave function on $[0,1]$ (if $d^{2} \psi(t) / d t^{2} \leqslant 0$ on $[0,1]$, then $\psi(t) \geqslant(1-t) \psi(0)+t \psi(1)$, and $\left.\psi(t) \leqslant d \psi(t) /\left.d t\right|_{t=0} \cdot t+\psi(0)\right)$ to the functions (B.10):

$$
\begin{aligned}
& \sigma_{2}^{+}(x, y) \geqslant(1-y) \sigma_{2}^{+}(x, 0)+y \sigma_{2}^{+}(x, 1), \\
& \sigma_{2}^{-}(x, y) \leqslant\left.\partial_{y} \sigma_{2}^{-}(x, y)\right|_{y=0} \cdot y+\sigma_{2}^{-}(x, 0) .
\end{aligned}
$$

Furthermore, the function $\sigma_{2}^{+}(x, 1)$ is concave, hence

$$
\sigma_{2}^{+}(x, 1) \geqslant(1-x) \sigma_{2}^{+}(0,1)+x \sigma_{2}^{+}(1,1) .
$$

Making use of the three inequalities just derived, and considering an explicit form of the function $f(x, y)$, we are led to the following estimate of $\sigma_{2}$ in $T$ :

$$
\sigma_{2}(x, y) \geqslant a^{*} \sigma_{0}-y\left(x K_{1}+K_{2}\right),
$$

where $\sigma_{0}$ is the entropy production in the initial state (10), and parameters $K_{1}$ and $K_{2}$ are given by

$$
\begin{aligned}
& K_{1}=\int \frac{f^{*}}{f_{0}}\left(f^{0}-f^{*}\right) d \Gamma+S_{B}\left(f^{0}\right)-S_{B}\left(f^{*}\right), \\
& K_{2}=\int \frac{f^{*}}{f_{0}}\left(f^{*}-f_{0}\right) d \Gamma+S_{B}\left(f^{*}\right)-S_{B}\left(f_{0}\right) .
\end{aligned}
$$

Here $S_{B}\left(f_{0}\right), S_{B}\left(f^{*}\right)$, and $S_{B}\left(f^{0}\right)$ are the values of the entropy in the states $f_{0}, f^{*}$, and $f^{0}$, respectively.

Since $\sigma_{0}$ is positive, there always exists a $y^{\prime}$, where $0<y^{\prime} \leqslant 1$, such that the RHS of (B.11) is non-negative for all $x$ in the segment [0,1]. More specifically, introduce a function $\varphi(x)=a^{*} \sigma_{0}-\left(x K_{1}+K_{2}\right)$, and denote

$$
z=a^{*} \sigma_{0} \min \left\{K_{2}^{-1},\left(K_{1}+K_{2}\right)^{-1}\right\}
$$

where $\min \left\{K_{2}^{-1},\left(K_{1}+K_{2}\right)^{-1}\right\}$ stands for the minimal of the two numbers, $K_{2}^{-1}$ and $\left(K_{1}+K_{2}\right)^{-1}$. Then $y^{\prime}$ is defined as

$$
y^{\prime}= \begin{cases}1 & \text { if } \varphi(x) \geqslant 0 \text { on }[0,1], \text { or } z \geqslant 1 \\ z & \text { otherwise. }\end{cases}
$$

Thus, $\sigma_{2}$ is non-negative inside the triangle $T^{\prime}=\overline{\operatorname{conv}}\left\{f_{0}, f_{1}\left(y^{\prime}\right), f_{2}\left(y^{\prime}\right)\right\}$, where $f_{1,2}\left(y^{\prime}\right)$ are given by (B.8), and $y^{\prime}$ is given by (B.14). If $y^{\prime}=1$, then $T^{\prime}=T$, and $\sigma_{2}$ is non-negative everywhere in $T$. In this case any pair of the functions $\xi(a)$ and $\eta(a)$ with the properties (B.5) and (B.6) give the approximation $f(a)$ consistent with the inequality (B.7). Otherwise, we continue the procedure. 


\section{B.3. Near-equilibrium estimations of the functions $\sigma_{1}$ and $\sigma_{2}$}

Let us come back to the specification (B.2) to establish the following inequalities for the functions $\sigma_{1,2}(\xi, \eta)$ (Eq. (B.4)):

$$
\begin{aligned}
& \sigma_{1}(\xi, \eta) \geqslant M_{1} \xi^{2}, \\
& \sigma_{2}(\xi, \eta) \geqslant M_{2} \xi .
\end{aligned}
$$

Inequalities (B.15) originate from the following consideration. Since $f(\xi, \eta) \rightarrow f^{0}$ as $\xi \rightarrow 0$, parameter $\xi$ controls a deviation of $f(\xi, \eta)$ from $f^{0}$ in $T$. Near the equilibrium state $f^{0}$, the function $\sigma_{1}(\xi, \eta)$ is quadratic in $\xi$, while the function $\sigma_{2}(\xi, \eta)$ is linear. Inequalities (B.15) extend these near-equilibrium estimations to other points of $T$, and they are intended to control a domination of $\sigma_{1}$ over $\sigma_{2}$ outside $T^{\prime}$ in the case $T^{\prime} \neq T$.

Writing $\sigma_{1}(\xi, \eta)=\xi \lambda(\xi, \eta)$ and representing $\lambda(\xi, \eta)$ as a combination of the concave functions, and after making the estimations as above, we obtain the following expression for $M_{1}$ in the first of the inequalities (B.15):

$$
M_{1}=S_{B}\left(f^{0}\right)-S_{B}\left(f^{*}\right) .
$$

Since $S_{B}\left(f^{0}\right)>S_{B}\left(f^{*}\right)$, expression (B.16) is always positive. An estimation of $M_{2}$ is much the same. Firstly, representing $\sigma_{2}(\xi, \eta)$ as in (B.10), and again estimating the concave functions obtained, we obtain the following inequality:

$$
\sigma_{2}(\xi, \eta) \geqslant \xi\left(\eta N_{1}+N_{2}\right),
$$

where the constants $N_{1}$ and $N_{2}$ are given by

$$
\begin{aligned}
& N_{\mathrm{I}}=\int \frac{f^{*}}{f^{0}}\left(f_{0}-f^{*}\right) d \Gamma+S_{B}\left(f_{0}\right)-S_{B}\left(f^{*}\right), \\
& N_{2}=\int \frac{f^{*}}{f^{0}}\left(f^{0}-f_{0}\right) d \Gamma+S_{B}\left(f_{0}\right)-S_{B}\left(f^{0}\right) .
\end{aligned}
$$

Secondly, denoting $\min \left\{N_{2}, N_{1}+N_{2}\right\}$ as the minimal of the two numbers, $N_{2}$ and $N_{1}+N_{2}$, we derive the constant in the second of the inequalities (B.15):

$$
M_{2}=\min \left\{N_{2}, N_{1}+N_{2}\right\} \text {. }
$$

As above, there are two possibilities:

1. If $M_{2} \geqslant 0$, then $\sigma_{2}$ is non-negative everywhere in $T$, and any pair of functions $\xi(a)$ and $\eta(a)$ with properties (B.5) and (B.6) gives $f(a)$ with the correct entropy behavior.

2. If $M_{2}<0$, then continue the procedure.

\section{B.4. Adjustment of the functions $\xi(a)$ and $\eta(a)$}

Let $y^{\prime}<1$ and $M_{2}<0$. A further analysis requires an explicit form of the functions $\xi(a)$ and $\eta(a)$ with the properties (B.5) and (B.6), and can be maintained in any specific 
case. Consider the simplest choice

$$
\xi(a)=1-a^{2}, \quad \eta(a)=g a,
$$

where $g, 0<g \leqslant 1$, is a parameter to be determined. The function (B.2) with the dependences (B.20) has a form (15):

$$
f_{g}(a)=f^{0}+\left(1-a^{2}\right)\left\{g a\left(f^{*}-f_{0}\right)+f_{0}-f^{0}\right\} .
$$

We should derive the parameter $g$ in (B.20) in such a way that (i) the states $f(a)$ of Eq. (15) belong to $T^{\prime}$, as $a$ varies from 0 to some $a_{1}$, and also that (ii) $\sigma_{1}(a)$ dominates $\sigma_{2}(a)$ as $a$ varies from $a_{1}$ to 1 . Under these conditions, the entropy inequality (B.7) is valid for all $a$ on the segment $[0,1]$.

Substitute now (B.20) into (B.7) and apply the inequalities (B.15) to get

$$
\frac{d S_{B}(a)}{d a} \geqslant 2 a\left(1-a^{2}\right) M_{1}-g\left(1-a^{2}\right)^{2}\left|M_{2}\right|
$$

We require that $f\left(a_{1}\right) \in \overline{\operatorname{conv}}\left\{f_{1}\left(y^{\prime}\right), f_{2}\left(y^{\prime}\right)\right\}$, and that the RHS of the inequality (B.22) is non-negative at $a_{1}$ :

$$
\begin{aligned}
& f_{g}\left(a_{1}\right)=f\left(x_{1}, y^{\prime}\right), \\
& 2 a\left(1-a^{2}\right) M_{1}-g\left(1-a^{2}\right)^{2}\left|M_{2}\right| \geqslant 0 .
\end{aligned}
$$

Here $f\left(x_{1}, y^{\prime}\right)$ is the specification (B.9) of the function $f_{g}\left(a_{1}\right)$. Explicitly, condition (B.23) reads

$$
\begin{aligned}
& a_{1}^{2}=x_{1} y^{\prime}, \\
& a_{1} g\left(1-a_{1}^{2}\right)=\left(1-x_{1}\right) y^{\prime}, \\
& g\left(1-a_{1}^{2}\right) \leqslant \frac{2 M_{1}}{\left|M_{2}\right|} a_{1} .
\end{aligned}
$$

Eliminating $a_{1}$ and $x_{1}$ in (B.24), we are left with the following estimation of the parameter $g$ :

$$
g \leqslant \lambda \frac{\sqrt{y^{\prime}(1+\lambda)}}{1-y^{\prime}+\lambda}
$$

where

$$
\lambda=\frac{2 M_{1}}{\left|M_{2}\right|}
$$

It may happen that the RHS of the inequality (B.25) is greater than 1. In this case we take $g=1$ in (B.21). Thus, if $y^{\prime}<1$ and $M_{2}<0$, the parameter $g$ in (B.21) and 
(15) is estimated as

$$
g=\min \left\{1, \lambda \frac{\sqrt{y^{\prime}(1+\lambda)}}{1-y^{\prime}+\lambda}\right\} .
$$

\section{B.5. Summary}

The choice of the parameter $g$ in the smooth approximation to the phase trajectory (15) is due to the following sufficient procedure:

1. Evaluate $K_{1}$ and $K_{2}$ (Eq. (B.12)).

2. If $a^{*} \sigma_{0}-\left(K_{1} x+K_{2}\right) \geqslant 0$ on $[0,1]$, take $g=1$, otherwise evaluate

$$
y^{\prime}=a^{*} \sigma_{0} \min \left\{K_{2}^{-1},\left(K_{1}+K_{2}\right)^{-1}\right\}
$$

3. If $y^{\prime} \geqslant 1$, take $g=1$, otherwise evaluate $N_{1}$ and $N_{2}$ (Eq. (B.18)).

4. If $\min \left\{N_{2}, N_{1}+N_{2}\right\} \geqslant 0$, take $g=1$, otherwise evaluate $M_{1}$ (Eq. (B.16)) and take

$$
g=\min \left\{1, \lambda \frac{\sqrt{y^{\prime}(1+\lambda)}}{1-y^{\prime}+\lambda}\right\}, \quad \lambda=\frac{2 M_{1}}{\left|M_{2}\right|} .
$$

The function $f_{g}(a)$ of (15) with $g$ thus derived has the following properties:

1. It begins in $f_{0}$ at $a=0$ and ends in $f^{0}$ at $a=1$.

2. It is a non-negative function of $\Gamma$ for each $a$.

3. It satisfies the conservation laws.

4. The entropy $S_{B}\left(f_{g}(a)\right)$ is a monotonic function of $a$.

5. It is tangent to the exact trajectory at $a=0$.

In practical calculations, the approximation $f_{1}^{*}=f_{0}+a_{1}^{*} Q_{0}$ with $a_{1}^{*}$ of (9) can be used elsewhere in this algorithm instead of the exact $f^{*}$.

\section{References}

[1] J. Dorfman and H. van Beijeren, in: Statistical Mechanics B, ed. B. Berne (Plenum, New York, 1977).

[2] A.V. Bobylev, Dokl. Akad. Nauk SSSR 225 (1975) 1041; 231 (1976) 571.

[3] M. Krook and T. Wu, Phys. Rev. Lett. 36 (1976) 1107; Phys. Fluids 20 (1977) 1589.

[4] M.H. Ernst and E.M. Hendriks, Phys. Lett. 70 A (1979) 183.

[5] E.M. Hendriks and M.H. Ernst, Physica A 120 (1983) 545.

[6] T. Carleman, Acta. Math. 60 (1933) 91.

[7] L. Arkeryd, Arch. Rat. Mech. Anal. 45 (1972) 1; 45 (1972) 17.

[8] C. Truesdell and R. Muncaster, Fundamentals of Maxwell's Kinetic Theory of a Simple Monatomic Gas (Academic Press, New York, 1980).

[9] M.H. Ernst, in: Studies in Statistical Mechanics. Vol. 10, eds. E. Montroll and J. Lebowitz (NorthHolland, Amsterdam, 1983).

[10] A.V. Bobylev, Sov. Sci. Rev. C 7 (1988) 111.

[11] A.N. Gorban, I.V. Karlin and V.B. Zmievskii, Modelling, Measurement and Control A 61(1) (1995) 29; 60 (4) (1995) 39.

[12] A.N. Gorban and I.V. Karlin, Transp. Theory. Stat. Phys. 23 (1994) 559.

[13] T. De Donder and P. Van Rysselberghe, Thermodynamic Theory of Affinity. A Book of Principles (Stanford Univ. Press, Stanford, 1936). 
[14] G.S. Yablonskii, V.I. Bykov, A.N. Gorban and V. Elokhin, Kinetic Models of Catalytic Reactions. Comprehensive Chemical Kinetics, Vol. 32 (Elsevier, Amsterdam, 1991).

[15] C. Cercignani, Theory and Application of the Boltzmann Equation (Scottish Acad. Press, Edinburgh and London, 1975).

[16] A.N. Gorban and I.V. Karlin, Physica A 190 (1992) 393.

[17] J.A. Tjon, Phys. Lett. A 70 (1979) 390.

[18] H. Cornille, J. Stat. Phys. 39 (1985) 181.

[19] A.N. Gorban, Equilibrium Encircling. Equations of Chemical Kinetics and their Thermodynamic Analysis (Novosibirsk, Nauka, 1984) [in Russian]. 